\title{
Continuing cancer care delivery during the peak of COVID-19 in the Bronx, New York: experience from a public teaching hospital
}

\author{
Surabhi Pathak ${ }^{1}$, Roshni Narurkar², Mohammed Hasan Khan ${ }^{3}$, Bei Jiang ${ }^{4}$, \\ May Nyein Chann Soe ${ }^{4}$, Caroline Hwang ${ }^{1}$, Monica Muppidi ${ }^{1}$, Wilbert S. Aronow ${ }^{3}$
}

\begin{abstract}
1Division of Hematology-Oncology, New York City Health and Hospital Corporation/ Lincoln Medical and Mental Health Center, the Bronx, New York, USA

${ }^{2}$ Division of Hematology-Oncology, Westchester Medical Center, New York Medical College, Valhalla, New York, USA

${ }^{3}$ Department of Cardiology, Westchester Medical Center, New York Medical College, Valhalla, New York, USA

${ }^{4}$ Department of Internal Medicine, New York City Health and Hospital Corporation/ Lincoln Medical and Mental Health Center, the Bronx, New York, USA
\end{abstract}

Submitted: 19 April 2021, Accepted: 30 April 2021

Online publication: 30 April 2021

Arch Med Sci 2021; 17 (4): 1109-1113

DOI: https://doi.org/10.5114/aoms/136299

Copyright $\odot 2021$ Termedia \& Banach

\begin{abstract}
Introduction: We report our experience with cancer care delivery during the peak of COVID-19 pandemic in New York City.

Methods: Retrospective analysis of the patients treated from the $1^{\text {st }}$ of March, 2020 to the $8^{\text {th }}$ of May, 2020.

Results: Team huddles, infection screening and patient selection strategies were implemented. One hundred and seventy patients were treated in 576 visits. Six developed severe COVID-19 requiring hospitalization, two died. Their median Charlson Comorbidity Index was 9, higher than the rest of the cohort.

Conclusions: Cancer care delivery is safe and feasible using an approach focused on careful patient selection, team communication and infection control.
\end{abstract}

Key words: COVID-19, cancer care delivery, team huddle.

Patients with cancer and those on active oncological treatment are a vulnerable group for COVID-19 with a high rate of mechanical ventilation and 30-day mortality [1-3]. Certain risk factors for cancer are also associated with worse outcomes in COVID-19, such as advanced age, high body mass index and chronic lung disease [3, 4]. Oncological treatments such as immunotherapy have been associated with worse outcomes among patients with COVID-19 [5].

Nevertheless, cancer care is time sensitive, and delays in delivery of oncological treatment are detrimental to its efficacy. Healthcare delivery including cancer care was affected during the COVID-19 pandemic secondary to patient fears, system constraints, resource diversion and challenges in balancing the risks of COVID-19 with the necessity of treatment of other medical conditions [6, 7].

New York City was the epicenter of the COVID-19 pandemic in March, 2020. Densely populated boroughs such as the Bronx reported the high-
Corresponding author: Surabhi Pathak New York Health and Hospital Corporation/ Lincoln

United States E-mail: surabhipathak@ gmail.com 
Table I. Measures implemented in the infusion center during the peak of the COVID-19 pandemic

\begin{tabular}{|l|}
\hline Staff and patient education \\
- Updates during twice daily huddle about the \\
ever-evolving information on the virus, its clinical \\
course, local criteria for testing and prevention \\
measures \\
- Patient education on social distancing, infection \\
precautions, guidance on testing and symptom \\
management \\
- Patients encouraged to call with any questions or \\
concerns \\
\hline Personal protective equipment (PPE) \\
- Face covering was initially optional, by mid-March \\
made mandatory \\
- Given the increase in admitted CovID-19 patients, \\
infusion staff were required to wear a respirator \\
and gloves for all patient interactions \\
- Staff were required to wear a gown and face \\
shield when accessing the chemotherapy port or \\
collecting a nasal swab for testing \\
- PPE use was monitored and strictly adhered to \\
SARS-2-COV-2 testing
\end{tabular}

- Given limited testing resources throughout the duration of the study, patients with symptoms and those requiring inpatient hospitalization were prioritized for testing

- Patients with cancer and history of exposure were also tested when feasible

Procedures for suspected infection

- All patients were called a day before the appointment for telephone screening for symptoms and infection exposure

- Additional screening prior to entry to the infusion center

- If symptomatic, the patient was isolated in a designated room followed by phone interview with a clinician and triaged to either outpatient testing or emergency room

- If chemotherapy was indicated the patient was treated in the isolation room

Selection of patients for oncological treatments

- All patients were reviewed during a team huddle

- Risk factors of severe COVID-19 and indications for continuing oncological treatment were discussed

- If risks outweighed the immediate benefit from treatment, patients were rescheduled to a later day and were informed during the telephone screening

Decrease risk of asymptomatic transmission

- Given limited testing resources, emphasis placed on preventing asymptomatic transmission, especially patient-to-patient

- Face covering was mandated for patients and staff

- Infusion chairs were separated by at least six feet

- Before and after use by the patient, infusion chairs were thoroughly sanitized

- Patients were advised to wash hands and use hand sanitizer before leaving the infusion center and the hospital est number of hospitalizations and were hotspots for infections, posing a challenge for care delivery for non-COVID-19 related medical issues [8]. During this time, the oncology infusion center at our hospital remained operational while we established new preventive safety measures for the infusion center and clinics as per professional oncological society guidelines. We report our experience managing patients at the outpatient infusion center during the peak of the pandemic and the effectiveness of our implemented preventive strategies.

Methods. Medical charts of the patients treated in the outpatient oncology infusion center at Lincoln Medical and Mental Health Center in the Bronx between March 1 and May 7, 2020 were reviewed. Details on demographic and clinical features, treatment details, co-morbid conditions, subsequent clinical course after infusion center visit were collected. Patients treated for rheumatological or endocrinological indications and those with incomplete information were excluded from the analysis.

De-identified data were analyzed using SPSS version 27 (IBM, Inc.). Descriptive analysis was used to tabulate clinical and demographic information. A multiple logistic regression model was applied to identify factors associated with COVID-19 among patients included in the study. The $p$-value of 0.05 was considered significant.

Results. A total of 170 patients were treated in 576 visits during the study period. Table I summarizes the measures taken in the oncology infusion center to mitigate the risk of COVID-19. Twice daily team huddles with pharmacists, medical oncologists, infusion nurses, medical assistants and social workers were implemented early in March and continued throughout the study period. Strategies focused on effective team communication, staff and patient education and infection control were adopted.

Table II lists the clinical and demographic features of the patients. Most of the patients had an Eastern Cooperative Oncology Group (ECOG) performance scale score of 0 or 1 . Breast cancer was the most common diagnosis (27.6\%), followed by gastrointestinal cancer $(17.6 \%)$ and lung cancer (14.7\%). Thirteen (7.6\%) patients with multiple myeloma, 5 (3\%) patients with lymphoma and 7 (4\%) patients with myeloproliferative neoplasms were treated. Five patients with non-malignant hematological conditions were also treated, including 1 patient with hemophagocytic lymphohistiocytosis, 1 with paroxysmal nocturnal hemoglobinuria, 2 with myelodysplastic syndrome and 1 with severe vitamin $B_{12}$ deficiency.

Of the 170 patients, 6 developed COVID-19 requiring hospitalization. Average age of patients 
requiring hospitalization was 63 years, 4 out of the 6 were female, and median time from infusion center visit to COVID-19 was 10.5 (6.2-23.7) days. Two patients had breast cancer, 1 patient had multiple myeloma, 1 patient had diffuse large B cell lymphoma, 1 patient had lung cancer and 1 patient had prostate cancer. Four patients were treated with cytotoxic regimens, 1 patient was treated with a lenalidomide-based regimen and another with leuprolide. The median Charlson comorbidity index $(\mathrm{CCl})$ score of these patients was 9, higher than the average for the rest of the cohort. Two of the 6 patients died due to COVID-19 and 3 made a complete recovery. One patient developed progression of lung cancer and was enrolled in hospice during hospital admission with COVID-19. There were 2 other patients with mild COVID-19 infection who were managed in the outpatient setting with supportive measures and had an uneventful recovery.

On multiple logistical regression analysis, history of diabetes was associated with increased risk of contracting severe COVID-19 (odds ratio 25.9 (95\% Cl: 1.3-519, $p=0.033)$ ). Other factors including age, gender, type of oncological treatment, smoking history, $\mathrm{CCl}$, type of cancer, growth factor support, nursing home residence, history of chronic kidney disease, history of coronary artery disease, statin use, and angiotensin converting enzyme inhibitor use were not associated with risk of developing severe COVID-19.

Discussion. Measures implemented in the infusion center as detailed above allowed for safe delivery of critical oncological care without a substantial increase in the risk of COVID-19 and its complications. The numbers of infections and deaths from COVID-19 observed in the study cohort appear compatible with the high rates of community transmission in New York City during the study period and cancer related risk for COVID-19. However, despite the preventive strategies, 6 patients in the study cohort developed severe COVID-19, of whom 2 eventually died. All the patients who developed severe COVID-19 had a high $\mathrm{CCl}$ score, underlining the importance of careful selection of patients for treatment and preventive measures, particularly during periods of high community transmission.

Team huddles have been associated with improvement in communication, coordination of care and reduction in errors [9, 10]. During the pandemic, twice daily team huddles allowed the team to review patient history and identify barriers for safe treatment delivery. It was an opportunity to review and discuss the dynamic hospital specific algorithm for COVID-19 and to prepare staff for a unified approach to patient care. Further examination of this potentially useful tool in
Table II. Clinical and demographic features of the patients treated in the infusion center

\begin{tabular}{|c|c|}
\hline Parameter & Results \\
\hline Total patients, $n$ & 170 \\
\hline Age [years], median (IQR) & $60.7(52-70)$ \\
\hline \multicolumn{2}{|l|}{ Gender, $n(\%)$ : } \\
\hline Male & $89(52)$ \\
\hline Female & $81(48)$ \\
\hline \multicolumn{2}{|l|}{ Race, $n(\%)$ : } \\
\hline Hispanic & $76(44)$ \\
\hline African American & $69(41)$ \\
\hline Caucasian & $10(6)$ \\
\hline Asian & $2(1)$ \\
\hline Others & $13(8)$ \\
\hline \multicolumn{2}{|l|}{ Insurance, $n(\%)$} \\
\hline Full Medicare/Medicaid & $38(22)$ \\
\hline Private health insurance & $79(47)$ \\
\hline $\begin{array}{l}\text { NY Emergency Medicaid/ } \\
\text { no insurance }\end{array}$ & $53(31)$ \\
\hline Charlson comorbidity scale, median (IQR) & $6.6(4-8)$ \\
\hline Nursing home residence, $n(\%)$ & $11(6.4)$ \\
\hline \multicolumn{2}{|l|}{ Primary diagnosis, $n$ (\%): } \\
\hline Solid organ malignancy & $134(79)$ \\
\hline Hematological malignancy & $31(18)$ \\
\hline Non-malignant etiology & $5(3)$ \\
\hline \multicolumn{2}{|l|}{ Intent of systemic therapy, $n(\%)$ : } \\
\hline Curative & $68(40)$ \\
\hline Palliative & $102(60)$ \\
\hline \multicolumn{2}{|l|}{ Systemic therapy, $n$ (\%): } \\
\hline Targeted therapy & $75(44)$ \\
\hline Immunotherapy & $10(6)$ \\
\hline Cytotoxic chemotherapy & $85(50)$ \\
\hline Prophylactic G-CSF given, $n$ (\%) & $34(20)$ \\
\hline Current or former smokers, $n(\%)$ & $81(47.6)$ \\
\hline Use of ACE inhibitor, $n(\%)$ & $59(35)$ \\
\hline Use of statins, $n(\%)$ & $69(40.5)$ \\
\hline $\mathrm{BMI}>25 \mathrm{~kg} / \mathrm{m}^{2}, n(\%)$ & $97(57)$ \\
\hline History of COPD, $n(\%)$ & $28(16.4)$ \\
\hline History of CAD, $n(\%)$ & $23(13.5)$ \\
\hline History of diabetes, $n(\%)$ & $54(31.7)$ \\
\hline History of hypertension, $n(\%)$ & $97(57)$ \\
\hline History of CKD, $n(\%)$ & $36(21)$ \\
\hline
\end{tabular}

oncological care delivery settings should be encouraged.

Diabetes mellitus is proposed as a risk factor for severe COVID-19; however, the magnitude of this association remains a matter of further study $[11,12]$. The effect of poorly controlled diabetes on immune response and associations with oth- 
er comorbid conditions such as metabolic syndrome are thought to heighten the risk of severe COVID-19 in this vulnerable population [11, 12]. In the current study, patients with a history of diabetes mellitus had higher odds of contracting severe COVID-19 requiring hospitalization. The impact of diabetes mellitus among patients with cancer and the risk of severe COVID-19 should be further evaluated to guide treatment decisions and additional precautions in this highly vulnerable group.

Receipt of immune checkpoint inhibitors has been suggested to be associated with poor outcomes with COVID-19 [5, 13]. In the current study cohort, there was no increased risk for COVID-19 among recipients of immune checkpoint inhibitors compared to other cancer treatments. Interactions between immune checkpoint inhibitors and the immune response to COVID-19 is an area of active investigation. While immunotherapy may not be a risk factor for infection with COVID-19, it might be associated with poor outcomes with COVID-19.

In the study cohort, there was no observed increased risk for severe COVID-19 relative to the type of cancer or kind of oncological treatments, whether cytotoxic chemotherapy, targeted therapy or immune therapy. Impact of cancer type and therapies on risk for severe COVID-19 is a matter of ongoing study. For example, UKCCMP and COVID-19 and Cancer Consortium found no increased risk for COVID-19 mortality related to receipt of specific cancer therapies, while a study from Memorial Sloan Kettering Cancer Center in New York City reported higher risk of severe COVID-19 among patients receiving immune checkpoint inhibitors [4, 11, 12].

The relatively small patient sample and data extraction using chart review are limitations of the current analysis. While the study sample is small, it is primarily composed of patients of African and Hispanic ethnicities, groups that have been associated with risk of severe COVID-19 and its complications $[14,15]$. The data provide insights into the safety of oncological care and potential risk factors for COVID-19 in this unique patient population. Importantly, the study highlights the efficacy of the preventive measures for protecting this vulnerable population from risks of severe COVID-19 while continuing critical oncological care.

In conclusion, cancer treatment in the outpatient setting using an approach focused on careful patient selection, infection prevention strategies and strong team communication is feasible even in the midst of the worst phase of a pandemic and allows for continuity of critical oncological care. This is more relevant because COVID-19 is expected to impact healthcare systems globally for the foreseeable future. Diabetes mellitus was associ- ated with high risk for COVID-19 related complications while receipt of cancer directed therapy and type of cancer were not associated with higher risk for infection compared to risks associated with community-based transmission. In communities with high community-based transmission, careful selection of patients for oncological based treatment is paramount.

\section{Acknowledgments}

We thank the pharmacy staff, infusion center nursing staff, medical assistants, ancillary staff and patients for making delivery of cancer care possible during these unprecedented times.

\section{Conflict of interest}

The authors declare no conflict of interest.

\section{References}

1. ElGohary GM, Hashmi S, Styczynski J, et al. The risk and prognosis of COVID-19 infection in cancer patients: a systematic review and meta-analysis. Hematol Oncol Stem Cell Ther 2020; S1658-3876(20)30122-9.

2. Kuderer NM, Choueiri TK, Shah DP, et al. Clinical impact of COVID-19 on patients with cancer (CCC19): a cohort study. Lancet 2020; 395: 1907-18.

3. Desai A, Sachdeva S, Parekh T, Desai R. COVID-19 and cancer: lessons from a pooled meta-analysis. JCO Glob Oncol 2020; 6: 557-9.

4. Scientific Evidence for Conditions that Increase Risk of Severe Illness|COVID-19|CDC [Internet]. [cited $2021 \mathrm{Apr}$ 18]. Available from: https://www.cdc.gov/coronavirus/ 2019-ncov/science/science-briefs/underlying-evidence-table.html?CDC_AA_refVal=https\%3A\%2F\%2Fwww.cdc.gov\%2Fcoronavirus\%2F2019-ncov\%2Fhcp\%2Fclinical-care\%2Funderlying-evidence-table.html

5. Robilotti EV, Babady NE, Mead PA, et al. Determinants of COVID-19 disease severity in patients with cancer. Nat Med 2020; 26: 1218-23.

6. Sud A, Jones M, Broggio J, al et. Collateral damage: the impact on outcomes from cancer surgery of the COVID-19 pandemic. Ann Oncol 2020; 31: 1065-74.

7. London JW, Fazio-Eynullayeva E, Palchuk MB, Sankey P, McNair C. Effects of the COVID-19 pandemic on cancer-related patient encounters. JCO Clin Cancer Informatics 2020; 4: 657-65.

8. COVID-19: Data Main - NYC Health [Internet]. [cited 2020 Nov 2]. Available from: https://www1.nyc.gov/site/ doh/covid/covid-19-data.page

9. Mazanec P, Arfons L, Smith J, et al. Preparing trainees to deliver patient-centered care in an ambulatory cancer clinic. J Cancer Educ 2015; 30: 460-5.

10. Page JS, Lederman L, Kelly J, Barry MM, James TA. Teams and teamwork in cancer care delivery: shared mental models to improve planning for discharge and coordination of follow-up care. J Oncol Pract 2016; 12: 1053-8.

11. Gupta R, Ghosh A, Singh AK, Misra A. Clinical considerations for patients with diabetes in times of COVID-19 epidemic. Diabetes Metab Syndr 2020; 14: 211-2.

12. Hussain A, Bhowmik B, do Vale Moreira NC. COVID-19 and diabetes: knowledge in progress Diabetes Res Clin Pract 2020; 162: 108142. 
13. Lee LYW, Cazier JB, Angelis V, et al. COVID-19 mortality in patients with cancer on chemotherapy or other anticancer treatments: a prospective cohort study. Lancet 2020; 395: 1919-26.

14. Millett GA, Jones AT, Benkeser D, et al. Assessing differential impacts of COVID-19 on black communities. Ann Epidemiol 2020; 47: 37-44.

15. Webb Hooper M, Nápoles AM, Pérez-Stable EJ. COVID-19 and racial/ethnic disparities. JAMA 2020; 323: 2466-7. 